\title{
Intermittent Preventive Treatment during Pregnancy with Sulphadoxine-Pyrimethamine May Promote Plasmodium falciparum Gametocytogenesis
}

\author{
F.A. Fehintola ${ }^{\text {b S.T. Balogun }}{ }^{\text {a }}$ S.B. Adeoye ${ }^{c}$ \\ a Department of Clinical Pharmacology and Therapeutics, College of Medical Sciences, University of Maiduguri, \\ Maiduguri, b ${ }^{b}$ Department of Pharmacology and Therapeutics, College of Medicine, University of Ibadan, and \\ ${ }^{c}$ Aremo Maternity Centre, Ibadan, Nigeria
}

\section{Key Words}

Intermittent preventive treatment $\cdot$ Malaria $\cdot$ Pregnancy

\begin{abstract}
Objectives: To determine the incidence of gametocytes and the propensity of sulphadoxine-pyrimethamine to promote gametocytogenesis when used as intermittent preventive treatment in pregnancy. Subjects and Methods: This observational non-interventional study assessed the influence of intermittent preventive treatment with sulphadoxine-pyrimethamine on Plasmodium falciparum gametocytaemia among Nigerian pregnant women. A total of 306 pregnant women were enrolled in the study. Results: The 306 pregnant women had 711 events, of which 31 pure gametocytaemic episodes were documented and 27 were recorded among the intermittent preventive treatment users. Only 4 episodes of pure gametocytaemia were recorded in pregnant women who did not receive any dose of sulphadoxinepyrimethamine $\left(27 / 129\right.$ vs. $\left.4 / 276, \chi^{2}=15.9, p=0.00006\right)$. Conclusion: Our findings show that intermittent preventive treatment in pregnancy with sulphadoxine-pyrimethamine may predispose to gametocyte carriage in pregnant women resident in the hyperendemic malaria region of southwest Nigeria. We therefore suggest that the use of insecticide-
\end{abstract}

treated nets be encouraged among pregnant women resident in malaria-endemic sub-Saharan Africa in order to reduce malaria transmission.

Copyright $\odot 2011$ S. Karger AG, Basel

\section{Introduction}

Children and pregnant women living in sub-Saharan Africa bear a disproportionate burden of malaria $[1,2]$. Women in their first or second pregnancy are known to be relatively more 'at risk' than multiparous women [3] and HIV-positive pregnant women have increased susceptibility [4-6]. Successful transmission and continued circulation of the malaria parasite requires ingestion of viable gametocytes by the female Anopheles mosquito during ingestion of blood. Profiling gametocyte carriage will aid efforts aimed at malaria control [7]. Since pregnant women have increased susceptibility to malaria infection [8-11], they would be expected to have a higher gametocyte carriage rate in an area hyperendemic for malaria.

Intermittent preventive treatment with sulphadoxinepyrimethamine is known to protect against the adverse consequences of malaria in pregnancy, thereby reducing

\section{KARGER}

Fax +4161306 1234

E-Mail karger@karger.ch

www.karger.com
(C) 2011 S. Karger AG, Basel

1011-7571/12/0211-0063\$38.00/0

Accessible online at:

www.karger.com/mpp
Dr. Fatai A. Fehintola

Department of Pharmacology and Therapeutics

College of Medicine, University of Ibadan

Ibadan (Nigeria)

Tel. +234 805520 2959, E-Mail fentolamine@yahoo.com 
the incidence of malaria episodes, malaria anaemia and poor obstetric outcome [11]. Based on the recommendation of the WHO, Nigeria, like other countries in subSaharan Africa, has adopted intermittent treatment with sulphadoxine-pyrimethamine in pregnancy, although access remains poor. Resistance to sulphadoxine-pyrimethamine is widespread and precludes its use as monotherapy; the drug also encourages gametocyte generation [12]. The primary objective of this study was to compare gametocyte carriage between pregnant women users and non-users of sulphadoxine-pyrimethamine as intermittent preventive treatment.

\section{Patients and Methods}

\section{Location of the Study}

This non-interventional observational study was conducted at a public secondary health facility in Ibadan, Oyo State, south-west Nigeria. Ibadan lies between $7.3^{\circ} \mathrm{N}$ latitude and $3.4^{\circ} \mathrm{E}$ longitude in the Guinea Savannah belt with an average rainfall of about 1,250 mm annually. Rainfall occurs from April through October each year, a period which coincides with the higher intensity of malaria transmission, which reflects the breeding rhythm of the Anopheles vector. The Research and Ethics Committee of the Oyo State Ministry of Health, Ibadan, approved the study, which lasted from January 2009 through July 2010. All consenting pregnant women attending the antenatal clinic were eligible for enrolment.

\section{Enrolment and Conduct of the Study}

Recruitment lasted from January 2009 to July 2010. Only confirmed pregnant women attending the antenatal clinic for the first time during the current pregnancy who consented were enrolled. All participants were interviewed by the investigators (F.A.F., S.B.A.) with a view to eliciting relevant obstetric and sociomedical history including malaria-preventive measures undertaken by them. Obstetric and other general examinations were carried out by the medical and nursing staff of the hospital as well as two of the investigators (F.A.F., S.B.A.). Upon enrolment and during subsequent visits, a physical examination was conducted and history recorded for each participant. During visits, particular note was taken of whether or not participants accessed intermittent preventive treatment in pregnancy with sulphadoxine-pyrimethamine (IPTp-SP) and relevant variables were consequently compared between the 2 'natural' groups. All the participants underwent the following tests: serology for $\mathrm{ABO}$ and Rhesus blood groups, syphilis as well as HIV status, according to the standard protocol used at public health facilities in Nigeria. In addition, thick and thin blood smears for quantification and speciation of malaria parasites were prepared from finger pricks following strict asepsis procedure. One or two drops of blood were spotted on filter paper for the purpose of PCR determination of parasite species and submicroscopic parasitaemia at a later date. Blood was collected into a heparinized capillary tube for determination of haematocrit using Hawskey ${ }^{\mathrm{TM}}$ Microhaematocrit centrifuge and read on the microhaematocrit reader. Subsequent visits of participants were governed by routine obstetric care of the hospital; for example, monthly visit until week 28 , biweekly visit between 28 and 36 weeks, and weekly thereafter until delivery.

\section{Screening and Quantification for Malaria Parasitaemia}

Screening and quantification for the malaria parasite followed the staining of air-dried thick smear using $10 \%$ freshly prepared Giemsa stain at $\mathrm{pH}$ 7.2. All slides were screened and quantified by a trained technologist and reviewed by either of 2 of the investigators (F.A.F./S.T.B.) who are skilled in basic malaria microscopy. No smear was declared negative until at least 100 oil immersion fields had been examined and whenever differences existed, the opinion of the third investigator became mandatory for a decision to be taken.

Quantification of Asexual and Sexual Forms of Plasmodium falciparum

Parasite density was determined on thick blood smear by counting all asexual and sexual stages against a minimum of 500 white blood cells or 500 asexual forms, whichever occurred first. Parasite density was thereafter obtained using the following formula, assuming a white cell count of $8,000 / \mu$ l of blood [13].

$$
\text { Parasite density }=\frac{\text { number of parasites } \times 8,000}{\text { number of white blood cells }}
$$

\section{Statistical Analysis}

The primary outcome measure was the presence of gametocytes in the peripheral blood films of the participants who attended the antenatal follow-up clinic while anaemia was considered as secondary outcome. Data obtained were fed into the SPSS version 15 statistical package comparing categorical parameters like presence or absence of gametocytes using the $\chi^{2}$ test while continuous variables such as age were compared using Student's t test. A p value of less than 0.05 was taken as statistically significant.

\section{Results}

\section{Sociobiological Characteristics and Peripheral Asexual Parasitaemia}

The total number of visits by the 306 women was 711 , while frequency of asexual malaria parasite positivity was 147 (20.7\%). All of the women attended 2 clinic visits, 81 were available for 3 visits, and 18 were seen 4 times. The mean number of clinic visits was $2.3 \pm 0.6$. The mean age was $26.7 \pm 5.1$ years and mean parity was $1.1 \pm 1.2(\mathrm{ta}-$ ble 1). Eighty-four (27.5\%) pregnant women accessed intermittent preventive treatment, whereas $222(72.5 \%) \mathrm{did}$ not.

Of the 306 women, 96 (31.4\%) were positive for asexual malaria parasite at the antenatal booking clinic. At subsequent clinic visits, that is, 2nd, 3rd and 4th, $14.7 \%$ (45/306), 7.4\% (6/81) and $16.7 \%(3 / 18)$ of the women were positive for malaria merozoites, respectively. The proportion of nulliparous women with peripheral asexual para- 
Table 1. Sociobiological characteristics of 306 pregnant women who accessed or did not access IPTp-SP

\begin{tabular}{|c|c|c|c|}
\hline & IPTp-SP & Non-IPTp-SP & $\mathrm{p}$ value \\
\hline Study participants & 84 & 222 & - \\
\hline \multicolumn{4}{|l|}{ Age, years } \\
\hline Mean \pm SD (range) & $27.6 \pm 5.6(18.0-40.0)$ & $26.4 \pm 4.9(16.0-38.0)$ & 0.307 \\
\hline \multicolumn{4}{|l|}{ Parity } \\
\hline Mean \pm SD (range) & $1.2 \pm 1.1(0.0-4.0)$ & $1.1 \pm 0.9(0.0-5.0)$ & 0.536 \\
\hline \multicolumn{4}{|l|}{ PCV at booking } \\
\hline Mean \pm SD (range) & $30.6 \pm 4.2(20.0-38.0)$ & $28.5 \pm 4.5(15.0-38.0)$ & 0.035 \\
\hline $\mathrm{PCV}$ at $3 \mathrm{rd}$ visit & $\mathrm{n}=36$ & $\mathrm{n}=45$ & \\
\hline Mean \pm SD (range) & $33.0 \pm 5(23-39)$ & $29.0 \pm 3.0(25-32)$ & 0.009 \\
\hline \multicolumn{4}{|c|}{ Proportion positive for asexual parasite } \\
\hline Booking & $14.3 \%(12 / 84)$ & $37.8 \%(84 / 222)$ & 0.0001 \\
\hline At follow-up ${ }^{1}$ & $0.0 \%(0 / 129)$ & $19.6 \%(54 / 276)$ & - \\
\hline \multicolumn{4}{|c|}{ Proportion positive for pure gametocyte } \\
\hline At booking & $0.0 \%(0 / 84)$ & $8.1 \%(18 / 222)$ & - \\
\hline At follow-up ${ }^{1}$ & $20.9 \%(27 / 129)$ & $1.5 \%(4 / 276)$ & 0.00006 \\
\hline
\end{tabular}

PCV $=$ Packed cell volume. ${ }^{1}$ Denominator includes participants who attended 2 nd, 3 rd, and 4 th visits.

sitaemia was significantly higher than that of multiparous women [57/135 (42.2\%) vs. 39/171 (22.8\%); $\mathrm{p}=0.004]$, although no difference was found in the mean asexual parasite density. At booking, 12 (14.3\%) of the 84 women in the IPTp-SP group were positive for malaria merozoites, whereas during subsequent visits, none had patent peripheral parasitaemia. On the other hand, 84 (37.8\%) of the 222 IPTp-SP nonusers were positive for the asexual forms at booking, and 45/222 (20.3\%), 6/45 (13.3\%) and $3 / 9(33.3 \%)$ were positive during the $2 \mathrm{nd}$, 3rd visit and 4 th visits, respectively.

Thirty-three women were positive twice such that 30 were positive at booking and at the 2nd visit, while 3 individuals were found to be positive at booking and at the 4 th visit. The sociobiological characteristics of women who were merozoite-positive on more than one occasion are summarized in table 2.

\section{IPTp-SP and Pure Gametocytaemia}

Pure gametocytaemia was documented in 18 women during the antenatal booking visit and in 31 others during subsequent visits. The mean number of gametocytes was 180 (range, 60-320/ $\mu$ l of blood). None of the IPTp-SP users had gametocytaemia at entry, but 27 of the 31 pure gametocytaemic episodes during the 2 nd through 4 th visits were recorded in women who had had at least 1 dose of sulphadoxine-pyrimethamine while the remaining 4 episodes involved pregnant women who did not receive any dose of sulphadoxine-pyrimethamine (27/129 vs.
Table 2. Sociobiological characteristics of women who were merozoite-positive on more than one occasion during follow-up

\begin{tabular}{ll}
\hline Parameter & Values \\
\hline Number & 33 \\
Age, years & $24.7 \pm 4.6(20.0-34.0)$ \\
PCV, \% & $27.5 \pm 4.0(22.0-34.0)$ \\
Number with PCV $<30 \%$ & $21(63.6 \%)$ \\
Gestation age, weeks & $22.2 \pm 5.8(16.0-32.0)$ \\
Gravidity & \\
$\quad$ Mean & $1.4 \pm 0.9(1-4)$ \\
$\quad$ Primigravidae & 27 \\
$\quad$ Secondigravidae & 3 \\
$\quad$ Multigravidae & 3 \\
Parity & $0.4 \pm 0.9(0.0-3.0)$ \\
ITN user & $3(9.1 \%)$ \\
IPT user & $0(0.0 \%)$ \\
\hline
\end{tabular}

PCV = Packed cell volume.

$4 / 276 ; \mathrm{p}=0.00006$ ). None of those with pure gametocytaemia used insecticide-treated nets (ITN) and their mean haematocrit value was significantly lower than that of the entire study population $[26.2 \pm 3.9(\mathrm{n}=30)$ vs. 29.2 $\pm 4.5 \%(\mathrm{n}=306), \mathrm{p}=0.0000001]$.

\section{Haematocrit (Packed Cell Volume and IPTp-SP)}

The mean haematocrit of all the women at booking was $29.2 \pm 4.5 \%$ and subsequently $30.4 \pm 4.6 \%(n=306)$, 
$31.0 \pm 4.5 \%(\mathrm{n}=81)$ and $30.3 \pm 5.9 \%(\mathrm{n}=18)$, respectively, at visit 2, 3 and 4. Although the haematocrit of IPTp-SP users was statistically higher than that of nonIPTp-SP users even at enrolment, the difference was more pronounced at subsequent visits; for example, the haematocrit at booking and at the 3rd visit were: $30.6 \pm 4.2$ versus $28.5 \pm 4.5 \%(\mathrm{p}=0.035)$, and $33.3 \pm 5.0(\mathrm{n}=36)$ versus $29.0 \pm 3.0 \%(\mathrm{n}=45)(\mathrm{p}=0.0009)$, respectively, for IPTp-SP users and non-users. During follow-up in the antenatal clinic, the increase of haematocrit for IPTp-SP users was statistically significant such that the haematocrit at entry was lower than at the 3rd visit [30.6 \pm 4.2 $(\mathrm{n}=84)$ vs. $33.3 \pm 5 \%(\mathrm{n}=36) ; \mathrm{p}=0.018]$. On the other hand, the haematocrit did not significantly increase in the IPTp-SP non-users during the same period [28.5 \pm $4.5(\mathrm{n}=222)$ vs. $29.0 \pm 3.0 \%(\mathrm{n}=45) ; \mathrm{p}=0.74]$.

\section{Discussion}

This observational study is probably the first attempt at profiling gametocytaemia in pregnant women who are exposed to IPTp-SP in our area of study. The sociobiological characteristics of the study population bear a resemblance to established epidemiology of malaria in the population of pregnant women resident in Nigeria. For example, a higher incidence of malaria was recorded among nulliparous compared to multiparous women with most cases present in the second trimester $[8,9]$. It is also an established fact that IPTp-SP reduces malaria merozoites, the incidence of malaria anaemia, and improves pregnancy outcome $[14,15]$. However, little or no attention has been directed at the propensity of sulphadoxine-pyrimethamine for the generation of gametocytes [12]. Reference must be made to the relatively poor access of malaria-preventive measures such as ITN and IPTp, both of which are known to be protective and have been recommended. That less than a third of the participants in this study were documented to have access to at least one dose of sulphadoxine-pyrimethamine and even poorer access to ITN is very worrisome.

Sulphadoxine-pyrimethamine has been documented to have a propensity for gametocyte generation when used in the treatment of acute uncomplicated malaria in children [11]. It has also been observed that gametocyte carriage in pregnant women resident in a malaria hyperendemic area is similar to the findings in children [16]. In the present study, it was observed that women IPTp-SP users had a higher incidence of gametocytaemia than IPTp-SP non-users with advancing pregnancy. The sig- nificance of this finding, if confirmed by larger studies, is that the use of sulphadoxine-pyrimethamine for IPTp though valuable in protecting adherents, may enhance malaria transmission and therefore defeat its own purpose in the long term. It is noteworthy that access to ITN was very low, thus further negatively impacting malaria control efforts as the female Anopheles mosquito vector may pick up viable gametocytes more easily and consequently transmit malaria. The fact that there is widespread high-grade resistance to sulphadoxine-pyrimethamine further complicates the matter, as it may mean that such circulating gametocytes are resistant to the drug. The result is that the use of sulphadoxine-pyrimethamine for presumptive treatment may be short-lived. Concerted efforts should therefore be directed at sourcing alternative drugs for IPTp. In addition, there is an urgent need to drastically improve access to ITN, particularly among pregnant women and young children who are resident in hyper-/holo-endemic malaria regions.

Although this non-interventional study involving only a few hundred participants may lack substantial power, therefore a limitation, it clearly suggests that adequate safeguards may yet be lacking in the IPTp-SP regimen and malaria control in sub-Saharan Africa. Larger epidemiological studies to address the subject of gametocytogenesis during IPTp-SP and gametocyte viability studies will be urgently needed as part of efforts aimed at reducing malaria morbidity and mortality.

\section{Conclusion}

This study suggests that IPT-SP may promote gametocyte generation when used by pregnant women residing in an area hyperendemic for malaria.

\section{Acknowledgement}

The authors acknowledge the support of all medical staff, especially the nursing staff at Aremo Maternity, Ibadan, Nigeria.

\footnotetext{
References

1 World Health Organization: World Health Statistics 2008: Mortality and Burden of Disease. Geneva, World Health Organization, pp 36-64.

-2 Menendez C, D’Alessandro U, ter Kuile FO: Reducing the burden of malaria in pregnancy by preventive strategies. Lancet Infect Dis 2007;7:126-135.
} 
-3 Steketee RW, Nahlen BL, Parise ME, Menendez C: The burden of malaria in pregnancy in malaria-endemic areas. Am J Trop Med Hyg 2001;64:28-35.

$>4$ French N, Nakiyingi J, Lugada E, Watera C, Whitworth JA, Gilks CF: Increasing rate of malarial fever with deteriorating immune status in HIV-1 infected Ugandan adults. AIDS 2001;15:899-906.

5 Whitworth J, Morgan D, Quiqley M, Smith A, Mayanja B, Eotu H, Omoding N, Okongo M, Malamba S, Ojwiya A: Effect of HIV and increasing immunosuppression on malaria parasitaemia and clinical episodes in adults in rural Uganda: a cohort study. Lancet 2000; 356:1051-1056.

-6 ter Kuile FO, Parise ME, Verhoeff FH, Udhayakumar V, Newman RD, van Eijk AM, Rogerson SJ, Steketee RW: The burden of coinfection with human immunodeficiency virus type 1 and malaria in pregnant women in sub Saharan Africa. Am J Trop Med Hyg 2004;71(suppl 2):41-54.

$>7$ Drakeley C, Sutherland C, Bousema TJ, Sauerwein RW, Targett GAT: The epidemiology of Plasmodium falciparum gametocytes: weapons of mass dispersion. Trends Parasitol 2006;22:424-430.
$>8$ Falade CO, Olayemi O, Dada-Adegbola HA, Aimakhu CO, Ademowo OG, Salako LA: Prevalence of malaria at booking among antenatal clients in a secondary health care facility in Ibadan, Nigeria. Afr J Reprod Health 2008;12:141-152.

$>9$ Nwagha UI, Ugwu VO, Nwagha TU, Anyaehie BU: Asymptomatic Plasmodium parasitaemia in pregnant Nigerian women: almost a decade after roll back malaria. Trans R Soc Trop Med Hyg 2009;103:16-20.

10 Enato EFO, Okhamafe AO, Okpere EE, Oseji FI: Prevalence of malaria during pregnancy and antimalarial intervention in an urban secondary care facility in southern $\mathrm{Ni}$ geria. Med Princ Pract 2007;16:240-243.

11 Menendez C, D’Alessandro U, ter Kuile FO: Reducing the burden of malaria in pregnancy by preventive strategies. Lancet Infect Dis 2007;7:126-135.

12 Sowunmi A, Fateye BA, Adedeji AA, Fehintola FA, Bamgboye EA, Babalola CP, Happi TC, Gbotosho GO: Effects of antifolates - cotrimoxazole and pyrimethamine-sulfadoxine - on gametocytes in children with acute, symptomatic, uncomplicated Plasmodium falciparum malaria. Mem Inst Oswaldo Cruz 2005;100:451-455.
3 Trape JF: Rapid evaluation of malaria parasite density and standardization of thick smear examination for epidemiological investigation. Trans R Soc Trop Med Hyg 1985; 79:181-184.

14 van Ejik AM, Ayisi JG, ter Kulle FO, Otieno JA, Misore AO, Odondi JO, Rosen DA, Kager PA, Steketee RW, Nahlen BL: Effectiveness of intermittent preventive treatment with sulphadoxine-pyrimethamine for control of malaria in pregnancy in western Kenya: a hospital-based study. Trop Med Int Health 2004;9:351-360.

15 Filler SJ, Kazembe P, Thigpen M, Macheso A, Parise ME, Newman RD, Steketee RW, Hamel M: Randomized trial of 2-dose versus monthly sulphadoxine-pyrimethamine intermittent preventive treatment for malaria in HIV positive and HIV negative pregnant women in Malawi. J Infect Dis 2006;194: 286-293.

16 Balogun ST, Fehintola FA, Adeyanju OA, Adedeji AA: Asexual and sexual stages of Plasmodium falciparum in Nigerian pregnant women attending booking clinic. $\mathrm{Ob}$ stetr Med 2010;3:106-109. 